\title{
Method to mitigate nystagmus and motion sickness with head worn visual display during vestibular stimulation
}

\begin{abstract}
Motion sickness and complaints of dizziness caused by provocative vestibular stimulating environments have been a widespread problem, affecting a significant portion of the world's population to varying degrees. The research objective was to determine if symptoms of motion sickness and associated nystagmus could be mitigated using an eye worn device which displayed an artificial horizon with a vertex point in the center for visual fixation, while experiencing vestibular stimulation. Assessment methods included an eye tracker, to measure velocity and duration of nystagmus, while patients underwent vestibular stimulation. This was followed by SSQ scoring to evaluate motion sickness symptoms. Users were tested with and without a visual display of specific symbology, while undergoing vestibular stimulation. Results demonstrated the associated nystagmus was significantly reduced in velocity and duration. With caloric stimulation testing, the duration of nystagmus was greater than 60seconds in the group without the visual display but decreased to 3.3 seconds when using the displayed symbology and instructing the user to focus on the vertex point between the artificial horizon and centered vertical line. Eye speed of nystagmus was diminished from 13.6degrees/second, to $0.4 \mathrm{degrees} / \mathrm{second}$ while using the visual display. Similar finding were noted with Barany Chair stimulation. The duration of nystagmus, which persisted for 25.8 seconds without the visual display, was decreased to 7.4seconds when using display and focusing on the vertex point. The eye speed of nystagmus was also decreased from 26.3 degrees/second to 5.7 degrees/second when using the visual display with vertex fixation. Objective SSQ scores were compared with and without visualization of the displayed symbology. When symbology was visualized, nausea, oculomotor symptoms, and disorientation were less apparent.
\end{abstract}

In conclusion, using an eye worn device which displays specific user orientation symbology can mitigate the symptoms of motion sickness and related nystagmus when experiencing vestibular stimulation.

Keywords: motion sickness, virtual environment, vestibular, visual display, nystagmus, eye fixation
Volume 7 Issue 5 - 2017

\author{
Wesley WO Krueger,' Frederick Bonato,' \\ Andrea Bubka ${ }^{2}$ \\ 'Montclair State University, USA \\ ${ }^{2}$ St Peters University, USA
}

Correspondence: Wesley WO Krueger, Montclair State University, 30 Haverhill Way, San Antonio, Texas, USA, Tel 210 854-203I,Email WkruegermdI@gmail.com

Received: May 22, 2017 | Published: June 14, 2017
Abbreviations: SSQ, simulator sickness questionnaire; VIMS, visual induced motion sickness; PTSD, post-traumatic stress disorder; OPN, optokinetic nystagmus; BPPV, Benign paroxysmal positional vertigo; HAS, head attitude scale; PRI, pitch/roll indicator; YI, yaw indicator; DoF, depth of field

\section{Introduction}

The impact of motion intolerance with resultant motion sickness is significant. It can result in incapacitating symptoms including nausea, emesis, unsteadiness, vertigo and loss of human performance. For those experiencing the symptoms, the result is often disabling with associated drowsiness, lack of concentration and disorientation. There are millions of people who annually experience motion sickness either because of vestibular stimulation or visually induced stimulation and the incidence is predicted to increase with increasing technology in new dynamic environments with advanced methods of locomotion and with the way we visualize information. ${ }^{1}$

Researchers report that up to $60 \%$ of the population have some motion intolerance. ${ }^{2}$ It is also known that some people are more susceptible than others; for example, women are more sensitive to motion than men by a ratio of about $5: 3 .^{3}$ Some are more susceptible due to physical reasons such as age. Studies show a significant genetic propensity to experience motion sickness. Susceptibility to motion sickness begins at about age two, and for most will peak in adolescence and decline gradually. However, many adults remain highly sensitive particularly when there is either an absence of a visual reference or exposed to significant levels of visual stimuli. In fact, a provocative visual stimulus has been shown to be the most influential cause of motion sickness symptoms. Reading in a moving vehicle, abruptly moving the head (such as looking down) while a vehicle is moving can provoke symptoms. Fear, anxiety and other psychological factors can contribute to the onset of motion sickness.

Seasickness, a common form of motion sickness, is frequent among naval personnel, where even $60 \%$ to $90 \%$ percent of inexperienced sailors can suffer from seasickness. ${ }^{4-7}$ This becomes a major problem in modern seamanship in which small crews are responsible for the operation of sensitive and sophisticated equipment. The occurrence of motion sickness can approach $100 \%$ in cruise ship passengers on rough seas. ${ }^{8}$

Airsickness complaints can vary up to $48 \%$ of passengers in commercial aircraft and can be very significant in military aerospace environments. ${ }^{9}$ In US Navy flight officers, $74 \%$ of student pilots experienced airsickness. ${ }^{10}$ Data from the Royal Air Force revealed $50 \%$ of aviators in high performance aircraft had airsickness. Symptoms appear to be more common when military personnel have no control of the aircraft. Symptoms of airsickness increased 
in more provocative environments with increased turbulence. ${ }^{11}$ In a questionnaire to B-1 and B-52 bomber crewmembers, it was reported to be a frequent occurrence among non-pilots in both aircraft, and experienced crewmembers were more likely to report an impact on their duties. ${ }^{12}$

Simulator sickness is another example of motion sickness, and many military pilots have reported at least one symptom following simulator exposure. ${ }^{13}$ Simulation sickness, or simulator sickness, is a condition where a person exhibits symptoms similar to motion sickness caused by playing computer/simulation/video games. In a study of Coast Guard aviators undergoing flight simulator testing, sixty-four percent $(64 \%)$ reported adverse symptoms during the first simulator flight and thirty-nine percent (39\%) did so during the last flight. ${ }^{14}$ The incidence of simulator sickness in non-pilots after virtual reality exposure is even greater, affecting approximating 60 to $80 \%$ of individuals. ${ }^{15}$ Thirty-Six percent $(36 \%)$ of pilots reported motion sickness when training on a Blackhawk flight simulator. ${ }^{16}$

Motion sickness can be induced, however, by either physical motion or visual stimuli which results in perceived motion, such as computer displays. ${ }^{17,18}$ Motion sickness due to virtual reality is very similar to simulation sickness and motion sickness due to films. In virtual reality, however, the effect is made more acute as all external reference points are blocked from vision, the simulated images are three-dimensional and in some cases stereo sound that may also give a sense of motion. Virtual reality is popular technology for entertainment purposes, and the virtual reality or augmented reality platform is an ideal system for military training applications and for post-traumatic stress disorder (PTSD) desensitization. Vision Induced Motion Sickness, is particularly prevalent when susceptible people are watching films on large screens such as IMAX but it may also occur in regular format theaters or even when watching TV. Solimini et al., ${ }^{19}$ reported a high occurrence of visually induced symptoms after watching $3 \mathrm{D}$ movies with $60 \%$ of individuals reporting 1 or more symptoms during the movie. ${ }^{19}$ IMAX and other panoramic type theaters often show dramatic motions such as flying over a landscape or riding a roller coaster. In the past, there has been no way to prevent this type of motion sickness except to close one's eyes during such scenes or to avoid such movies. In these cases, motion is detected by the visual system and hence the motion is seen, but no motion or little motion is sensed by the vestibular system. Viewing 3D movies can increase nausea, oculomotor findings and disorientation.

Vection has been found to be correlated with levels of visual induced motion sickness (VIMS) and postural status. Vection can be defined as a sensory-spatial illusion that creates false sensations of self-motion, in either linear or angular directions. However, several recent studies suggest that vection induction does not require explicit motion. ${ }^{20}$ The correlation between vection and VIMS has led to the term vection induced motion sickness. ${ }^{21,22}$ Visually induced vection can be quite compelling, and the illusion has been investigated extensively for over a century. Although false perceptions of self-motion are common, visual characteristics linked to this type of illusion are not fully understood. Vection can be strongly influenced by various physical aspects. Rotating auditory cues can also induce vection but auditory vection is much weaker and far less compelling than visual vection, which can be indistinguishable from real motion..$^{23}$ Most people know the phenomenon of vection from real-world experience. When sitting in a train waiting to depart from the train station and watching a train on the neighboring track pulling out of the station, one can have the strong impression of moving oneself, even though it was in fact the train on the nearby track that just started to move. A similar effect can be observed when sitting in a vehicle waiting for the traffic light to turn green and viewing a nearby vehicle slowly staring to move. It has been consistently reported that a visual stimulus encompassing a wider visual field induces stronger vection ${ }^{24}$ and that the peripheral visual field is more effective for vection induction than is the central visual field. Regardless of the type of sickness experienced, it is apparent that the leading cause of symptoms is a sensory mismatch, and specifically there is a discrepancy between the inner ear, vision and proprioceptive responses to some stimulus. Whatever type of stimulation has caused the symptoms, in severe motion provocative environments, motion sickness results in loss in human performance affecting cognitive and motor skills.

It is also apparent that visual input is necessary for compensation with vestibular system stimulation and the use of visual information may reduce symptoms significantly. Stern et al., ${ }^{22}$ reported that eye fixation reduced motion sickness and Webb and Griffin similarly supported this finding by demonstrating that motion sickness symptoms were reduced when subjects focus on a stationary cross in an optokinetic drum environment. ${ }^{25}$ Eye fixation has consistently been shown to significantly reduce levels of visually induced motion sickness (VIMS) and has been was associated with the suppression of eye movement. What has been lacking in the literature is a study which correlates the nystagmus with motion sickness symptoms, while using an effective drug free methodology to reduce both the nystagmus and symptoms during vestibular stimulation.

In the present study, a see-through eye worn device displayed user orientation information while the individual being tested underwent vestibular stimulation. The symbology viewed by the user was comprised of an artificial horizon with a vertex point in the center for visual fixation. User activity of pitch, roll and yaw movement was also visualized. The goal was to determine the efficacy of using such a display device with this symbology for mitigating the symptoms of motion sickness/disorientation and correlate these findings with the velocity and duration of the associated nystagmus.

Specifically, the lateral semicircular canal was stimulated using cold calorics and Barany Chair rotation, conditions known to lead to motion-induced symptoms. Objective eye tracking was performed to correlate the motion sickness symptoms with the actual eye movements, while the vestibular system was stimulated. It was hypothesized that visual fixation on the specific orientation symbology described would reduce the nystagmus as well as the motion sickness and disorientation symptoms in a provocative environment when the vestibular system was being stimulated.

\section{Materials and methods}

Eighteen patients voluntarily participated in the study ( 6 men, 12 women) (mean age $=45.4$ years). Ten (10) patients were previously diagnosed with motion sickness, five had labyrinthine dysfunction, and three were diagnosed with benign paroxysmal positional vertigo (BBPV). All patients had some degree of motion sensitivity. The occurrence of symptoms was typically variable.

The two methods of vestibular stimulation used included caloric irrigation and Barany Chair rotation. For each individual being tested, the see-through glass used for display of the symbology was worn under the eye tracking system (provided by Visual Eyes goggles from Micromedical Technologies ${ }^{\mathrm{TM}}$ Inc.). During the testing, the eye movements were tracked in the darkened Visual Eyes TM goggles). In one of the lenses (e.g. right lens) either the user centric symbology discussed was illuminated and visualized (as "enabled mode") or not 
visualized (as "disabled"). When turned on, several visual components were visible through the right lens of the display. These components included:

a. A Head Attitude Scale (HAS) which is a magenta-colored Cartesian graph with equal-length $\mathrm{x}$ - (horizontal) and $\mathrm{y}$-(vertical) axes.

b. A Pitch/Roll Indicator (PRI)-a tangerine-colored line with a length approximately one-third the length of the HAS horizontal and is bisected by, moves vertically along, and rotates (tilts) about the HAS vertical axis.

c. A Yaw Indicator (YI) that is a tangerine-colored, open-ended, three-sided trapezoid that "slides" the length of the PRI as the wearer's head is rotated left or right. Each symbol in the display has a specific function. The presence of the HAS artificial horizon line can reduce the effects of the pitch and roll motion to the user and can increase cognitive task performance by lessening the visual-vestibular conflict. This pitch and roll indicator line can confirm what the inner ear and proprioception have sensed when there is no or limited visual reference, such as in darkness, or the visual information is misleading. It aids proprioception by visual confirmation of head and body location and movement.

All patients used the displayed symbology, as seen in (Figure 1), to maintain orientation and provide themselves with a specific point of visual fixation. Each subject had vestibular stimulation with the visual symbology disabled (e.g. illuminated visual display turned off) and enabled (e.g. illuminated visual display turned on). The symbology was randomly selected for each trial but each patient had ultimately four (4) tests (e.g. caloric irrigation with and without the fixation symbology, as well as Barany chair rotation with and without the fixation symbology). Each subject served in both the experimental and control conditions. All patients had no labyrinthine suppressants or labyrinthine stimulation for 72 hours prior to testing.

In the right eye of the lens, when enabled, the patient was visualizing (Figure 1). The symbology was designed to provide the user with information regarding his or her positional orientation as well as an artificial horizon and central vertex point (for focused visual fixation) on the HAS vertex symbol. A video engine displayed the image provided below with 3 axes $(6 \mathrm{DoF})$ positional sensing. This would sample multiple head movements each second. The symbology components were comprised of an artificial horizon with centered vertical axis, a pitch and roll indicator, and a yaw indicator. Normally the artificial horizon and vertical axis remained stationary, but the pitch, roll and the yaw indicators had the ability to move, as the patient moved. In this testing environment, the head was fixed in a stationary position and no movement was therefore present with the pitch, roll or yaw indicators.

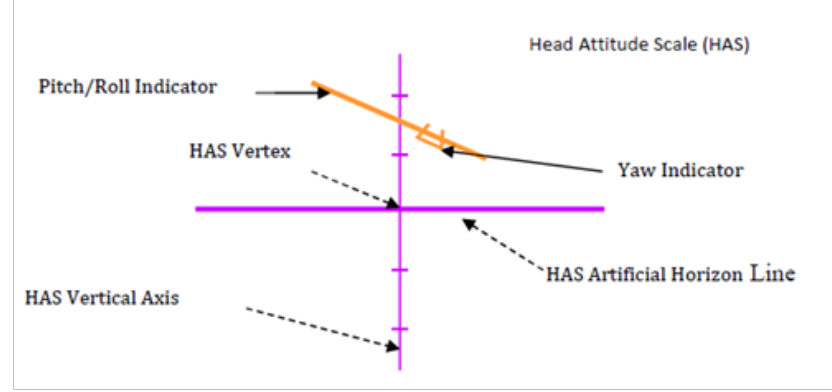

Figure I Symbology components of the displayed technology.
As they received vestibular stimulation, individuals being tested were instructed to focus on the central fixation point of the symbology components (e.g. at the junction of the vertical and horizontal axis or HAS vertex) throughout the trial. The specific purpose was to abort the abnormal eye movements or nystagmus. In the control condition (e.g. the technology was "disabled"), the subject was instructed to simply keep the eyes open and look into the dark goggles as they were undergoing testing.

Cold air irrigation $\left(16.2^{\circ} \mathrm{C} / 61.16^{\circ} \mathrm{F}\right)$ for 60 seconds was used and the duration of the nystagmus post-stimulation was measured as well as the average slow component velocity nystagmus, without and without the technology for 60 seconds following the stimulation. The measurement for 60 seconds was arbitrarily chosen as the nystagmus normally will decline by this time with caloric irrigation. The test subject was supine with the head inclined 30degrees up from the horizontal plane to make the lateral canal horizontal. The average slow component velocity nystagmus was measured in degrees/sec.

A customized Barany chair was utilized and assessment was performed with a peak velocity chair rotation of 160-180degrees/ second without and with the technology. This was done in the head up right, chin tilted downward 20 degrees to maximize the lateral SSC stimulation. The subjects were rotated for 30 seconds and although the recording began at the onset of rotation, measurements were taken when the chair stopped. Similarly, the average slow component velocity nystagmus was measured as well as the duration of the nystagmus during the post-stimulation period for 60 seconds.

All the patients were also assessed using the Simulator Sickness Questionnaire (SSQ) ${ }^{17}$ and provided subjective helpfulness ratings at the conclusion. When scored according to published guidelines ${ }^{18}$ the SSQ yields four scores: a total SSQ score and three sub-scores corresponding to nausea, oculomotor symptoms, and disorientation. Patients indicated the level at which each symptom was experienced, both pre-treatment and post-treatment. Patients were also asked to rate their overall sickness level after each trial using a 0-10 overall wellbeing scale $(0=\mathrm{I}$ feel fine, $10=\mathrm{I}$ feel awful as if $\mathrm{I}$ am about to vomit). Additionally, subjects rated self-motion perception using a 0-10 scale. Test results and SSQ scores were analyzed using the Student t-test for dependent measures. Differences were considered be significant at an alpha level of 0.05 .

\section{Results and discussion}

\section{Eye tracking results with caloric irrigation and videonystagmography}

The mean duration of nystagmus with caloric stimulation demonstrated nystagmus persisting more than 60 seconds when the symbology was not visible ("disabled), but when visible ("enabled") the mean duration of nystagmus was only 3.3 seconds $t(17)=17.00$, $\mathrm{p}<.0001$. The mean eye speed was measured at 13.06 degrees/second without the visible symbology ("disabled") and 0.4 degrees/second with the use of the symbology ("enabled") $\mathrm{t}(17)=7.43, \mathrm{p}<.0001$.

\section{SSQ for caloric testing}

SSQ scores were obtained in the Caloric testing experimental condition (symbology "enabled") were significantly lower (significance defined as $\mathrm{p}<.05$ two-tailed) than those obtained in the control condition (symbology "disabled). Four scores were calculated for each subject: a total SSQ score and three sub-scores corresponding to nausea, oculomotor symptoms, and disorientation. For the 
experimental conditions, dependent t-tests revealed significantly lower total SSQ scores $t(17)=2.33, p=0.032$, and lower sub-scores corresponding to nausea $\mathrm{t}(17)=2.36, \mathrm{p}=0.030$, oculomotor symptoms $\mathrm{t}(17)=1.60, \mathrm{p}=0.128$, and disorientation $\mathrm{t}(17)=2.30$, $\mathrm{p}=0.034$.

When the patients were asked to indicate the effect of the display as to whether they experienced motion sickness symptoms the mean rating was 6.11 (where $7=$ greatly reduced, $6=$ much reduced, $5=$ some reduced, $4=$ not changed, $3=$ some increased, $2=$ much increased, 1=greatly increased). All 18 patients reported that overall results in mitigating the symptoms and lessening vection were better with visualization of the display.

\section{Eye tracking results with barany chair and videonystagmography}

The mean duration (seconds) of nystagmus with Barany Chair stimulation demonstrated nystagmus persisting 25.8 seconds when the symbology was not visible ("disabled), but when visible ("enabled") the mean duration of nystagmus was only 7.4seconds $t(17)=7.3$, $\mathrm{p}<.0001$. The mean eye speed (degrees/second) with Barany Chair stimulation was measured at 26.3 degrees/second without the visible symbology ("disabled") and 5.7 degrees/second with the use of the symbology ("enabled") t (17) $=10.81, \mathrm{p}<.0001$.

\section{SSQ for barany chair testing}

SSQ scores were obtained in the Barany chair experimental condition (symbology "enabled") were significantly lower (significance defined as $p<.05$ two-tailed) than those obtained in the control condition (symbology "disabled). Similarly, four scores were calculated for each subject using methods and weighting factors as previously noted with: a total SSQ score and three sub-scores corresponding to nausea, oculomotor symptoms, and disorientation. For the experimental conditions, dependent t-tests revealed significantly lower total SSQ scores $\mathrm{t}(17)=3.95, \mathrm{p}=0.001$, and lower sub-scores corresponding to nausea $\mathrm{t}(17)=3.61, \mathrm{p}=0.001$, oculomotor symptoms $\mathrm{t}(17)=3.20, \mathrm{p}$ $=0.004$, and disorientation $\mathrm{t}(17)=3.04, \mathrm{p}=0.005$.

The patients were asked to rate the effectiveness using the visualized displayed symbology for lessening the vertigo/motion sensation/vection during and after chair rotation and the mean rating was 6.06 and 6.0 respectively (where $7=$ greatly improved, $6=$ much improved, $5=$ some improved, $4=$ not changed, $3=$ some increased, 2 $=$ much increased, $1=$ greatly increased). None of 18 patients reported any adverse side effects experienced with the display (headache and fullness of the head). Like those results seen with caloric stimulation, all 18 patients reported better results while visualizing the displayed symbology during vestibular stimulation.

Following the completion of both the caloric and Barany chair testing, the patients were then asked to rate how using the displayed symbology improved or worsened their symptoms and sensation of vection. The mean rating was 6.22 (where $7=$ greatly improved, $6=$ much improved, 5=some improved, 4=not changed, 3=some increased, $2=$ much increased, $1=$ greatly increased).

Prior NIH studies have previously documented the feasibility and efficacy of using a head worn see-through visual display for real life use in motion provocative environments (such as in air, boat and auto travel) to mitigate motion sickness. ${ }^{26}$ The patients used the display in everyday life situations, known previously to elicit their symptoms. Specifically, in the phase I study $85 \%$ of those patients who specifically had motion sickness rated the visually displayed symbology as positive in controlling their symptoms. In the phase II study, $100 \%$ of patients rated the technology as being extreme to moderately helpful in controlling the motion sickness symptoms, while performing out of the laboratory motion provocative activities. Notably when using such a visual display, there was significant mitigation of emesis and the duration of motion sickness symptoms was significantly shorter. A study at Saint Peter's University demonstrated the value of visual fixation, using this symbology, on diminishing the vision induced motion sickness symptoms while in an optokinetic drum ${ }^{27}$. In this study, all the overall SSQ scores were significantly lower when the subjects were focusing on the eye worn displayed symbology.

In these noted studies, the patients had visual or vestibular stimulation either in real life circumstances or in the laboratory and the findings agreed with those obtained in other studies which demonstrated that visual fixation on specific symbology reduced the magnitude of motion sickness symptoms. ${ }^{25}$ However, no eye tracking of abnormal eye movement was performed to correlate the symptoms or findings. When subjected to visual or vestibular stimulation individuals often have abnormal eye movement resulting in the complaints of disorientation. When the stimulus is significant, nystagmus will result. Other studies have documented the presence of nystagmus with individuals with motion sickness. Attias et.al correlated the intensity of nystagmus during sea sickness episodes on a vessel and determined the nystagmus was episodic, direction-fixed and its intensity was greater in sea-sick than in non-sick subjects. ${ }^{28}$ Their findings suggested that the nystagmus recorded is labyrinthine in origin and stems from temporary peripheral vestibular dysfunction elicited by sea sickness. Bukhtiiarov, et.al demonstrated that interocular nystagmus asymmetry is linked directly with the severity of motion sickness symptoms. ${ }^{29}$

Nystagmus is an involuntary oscillation of one or both eyes about one or more axes. Generally, the eyes move rapidly in one direction (fast phase or fast component) and slowly in another direction (slow phase or slow component). Individuals experiencing nystagmus usually have decreased vision and poor depth perception.

Nystagmus patients often experience fluctuations in their vision. A change in the speed of the nystagmus leading to a decrease in vision can be related to stress, the patient's emotional state, fatigue, the direction of view and to the intensity of the stimulus (visual or vestibular). Patients with nystagmus also report problems with balance. Impairment to binocular vision is common with early onset nystagmus and depth perception is indirectly impaired in many patients.

Nystagmus occurs during self-rotation to hold images of the visual world steady on the retina and maintain clear vision. Two forms of nystagmus are induced by self-rotation: optokinetic and vestibular. An optokinetic nystagmus is an involuntary, conjugate, jerk nystagmus that is seen when a person gazes into a large moving field. The oscillations, which are in the plane of the moving field, are generally $3-4^{\circ}$ in amplitude and $2-3 \mathrm{~Hz}$ in frequency. Both cortical and subcortical pathways contribute to the response, which is driven by the retinal image slip velocity.

Vestibular nystagmus occurs during rotation. The inner ear contains motion detectors (e.g. vestibular labyrinth) that project to the vestibular nuclei and cerebellum. Vestibular nystagmus can also be induced by irrigating the ears with warm or cold water or air. With unilateral irrigation, the conjugate nystagmus is horizontal, vertical, torsional or oblique, depending on the position of the head. 


\section{Control mechanisms for maintaining steady gaze- fixation include}

a. The vestibulo-ocular reflex.

b. A gaze-holding system (the neural integrator) that operates whenever the eyes are required to hold an eccentric gaze position. Failure of any of these control systems will bring about a disruption of steady fixation. Two types of abnormal fixation can result-nystagmus and saccadic intrusions/oscillations. The essential difference between them lies in the initial movement that takes the line of sight off the object of regard. In the case of nystagmus, it is a slow drift or 'slow phase' often due to a disturbance of one of the three mechanisms for gaze stability. On the other hand, with either saccadic intrusions or saccadic oscillations, an inappropriate fast movement moves the eyes off target.

Nystagmus may decrease when the eyes converge to read. Fixation overrides the vestibular stimulus and results in suppressing both preand post-rotational nystagmus. This proves that the foveal vision and not peripheral vision is responsible for the suppression by fixation. ${ }^{30}$

Eye fixation has consistently been shown to significantly reduce levels of visually induced motion sickness (VIMS). The common belief is that the reduction in VIMS is associated with the suppression of abnormal eye movement. There is a clear correlation between the percentage reduction of suppression in slow-phase velocity and amplitude during the period of eye opening and ocular fixation. ${ }^{31}$

When subjects look at a target stabilized at the fovea presented against sinusoidal motion of an optokinetic stimulus field, optokinetic nystagmus $(\mathrm{OKN})$ is suppressed. Increased visual demand modifies the nystagmus waveform favorably (and possibly adaptively), producing a significant reduction in nystagmus intensity and prolonged foveation 32,33 . Individuals can suppress caloric nystagmus with a visible target very well, but they can only partially suppress caloric nystagmus with an imaginary target and with after-image. ${ }^{34}$

The amplitudes of nystagmus reactions, evoked by sinusoidal harmonic acceleration, can be quantitatively suppressed by visual fixation. The degree of suppression depends on age. The trend to better fixation suppression increases up to the end of the age of forty. The ability to suppress vestibular nystagmus decreases, however, after reaching the fortieth year of life.

\section{Conclusion}

Having a stable visual input has been the key to control motion sickness and spatial disorientation. The current study has been a follow-on evaluation using an eye worn see-through visual display which has been designed to control the objective findings of motion sickness and symptoms to provide better orientation and therefore enhance the human performance when in a provocative motion environment. It is known that foveal vision is primarily responsible for this and it can modify the nystagmus by both reducing the intensity and duration of nystagmus. Anytime nystagmus is present there will difficulty focusing on a target of interest, cognitive difficulty and loss of human performance. Multi-tasking and performance has been shown to deteriorate while experiencing mild motion sickness. ${ }^{35}$

This current study establishes the fact that fixation of the specific displayed user oriented symbology with artificial horizon can override the vestibular stimulation and stabilize the eye reflexes and therefore aid target acquisition. Although foveal fixation on the HAS vertex, as seen in (Figure 1), is extremely important when experiencing rotation or vertigo, the peripheral vision will visualize on the HAS artificial horizon line in a slightly "defocused" manner. Although focusing on a laser spot has been shown to suppress nystagmus during caloric or optokinetic testing, subjects in this study did not prefer just to look at a "dot" or central axis point only but preferred to focus on the HAS vertex with the HAS artificial horizon line. If only a dot was available to suppress the nystagmus, there will be a minimum "dot" size required to suppress nystagmus and this size will be correlated with subjective visual acuity.

Fixation greatly reduced nystagmus, with respect to duration and velocity, when the labyrinth was stimulated. This correlated with mitigation of symptoms of motion sickness and reduced sensation of vection, as documented with the SSQ. Users expressed the additional value of the artificial horizon line as a stable point of reference and it seems to have more value in those having motion sickness symptoms than just experiencing violent vertigo. Specifically, during violent vertigo users focused on the HAS vertex, because the foveal vision controlled the symptoms most, but with a rolling motion sensation most users will prefer to look at the HAS vertex and HAS artificial horizon line.

The use of an eye tracker to measure eye movement is of benefit in the evaluation of the symptoms of motion sickness, spatial disorientation and vection. The presence of nystagmus not only can be correlated with the symptoms but the velocity and duration appears to be directly related (e.g. the greater velocity and duration is a correlate to increased motion sickness symptoms and sense of vection). The mitigation of the nystagmus, both in duration and velocity can also be seen with the improvement in the symptoms of motion sickness and in vection (although to a lesser degree than the symptoms). In this study with calorics and Barany chair vestibular stimulation, the nystagmus velocity (e.g. eye speed in deg./sec.) and duration (e.g. sec.) were significantly reduced and the symptoms were mitigated while using the visual display system. When the symptoms of motion sickness are mitigated and the objective findings of nystagmus can be controlled, the human performance to attend to other tasks can be improved.

In summary, this study demonstrated the efficacy of alleviating symptoms of motion sickness and vection while wearing a seethrough eye worn display, which projected an artificial horizon with a vertex point in the center for visual fixation, when experiencing vestibular stimulation. Although previous studies have used this visual display in everyday and practical situations or in visually stimulating environments, this study used an eye tracker to correlate the symptoms of motion sickness and vection with eye movement and demonstrated the effectiveness of visualizing this display in mitigating the symptoms as well as suppressing nystagmus velocity and duration. The eye worn display system described is unique in motion provocative environments, as it is user centric as opposed to being vehicular centric and can be used in any type of eye worn visual display system, such as with Google Glass or in virtual or augmented platforms.

The intent of the displayed symbology is to keep the user oriented and symptom free, in a vestibular stimulating environment, to complete necessary tasks. It has use in any vehicular travel, when exposed to adverse travel circumstances and can be used in mitigating symptoms of dizziness in the rehabilitation environment. When worn, the user is not constantly focusing on the symbology, but only when in a visual or vestibular stimulating situation. Having the display in an off-bore position (e.g. not in the center of the visual field) would also enable the user to continue their activities without distraction or 
visual clutter. Additionally, this disruptive technology can be used in virtual reality or augmented reality platforms for gaming experiences, entertainment, occupational or military training and desensitization. When used in these platform systems, it has been shown to prevent motion sickness/disorientation when the user is exposed to provocative visual stimuli and enable the user to wear the platform system for longer periods.

\section{Acknowledgments}

None.

\section{Conflicts of interest}

Author declares there are no conflicts of interest.

\section{Funding}

None.

\section{References}

1. McCauley ME, O'Halion JF, Royal JW, et al. Motion Sickness Incidence: Exploratory Studies of Habituation, Pitch and Roll, and the Refinement of a Mathematical Model. Human Factors Research, Inc. Office of Naval Research, Virginia, USA. 1976. p.1-61.

2. Benson AJ. Motion Sickness. Encyclopaedia of occupational health and safety. (4th edn), International Labour Office, Geneva, USA. 1998. p.12-14.

3. Dobie TG, May JG. Cognitive-behavioral management of motion sickness. Aviat Space Environ Med. 1994;65(10 Pt 2):1C-C2.

4. Lawther A, Griffin MJ. A survey of the occurrence of motion sickness amongst passengers at sea. Aviat Space Environ Med. 1988;59(5):399-406.

5. Chan G, Moochhala SM, Zhao B, et al. A comparison of motion sickness prevalence between seafarers and non-seafarers onboard naval platforms. Int Marit Health. 2006;57(1-4):56-65.

6. Turner M, Griffin MJ. Motion sickness in public road transport: passenger behavior and susceptibility. Ergonomics . 1999;42(3):444-461.

7. Stevens SC, Parsons MG. Effects of motion at sea on crew performance: A Survey. Marine Technology Society. 2002;39:29-47.

8. Gahlinger PM. Cabin location and the likelihood of motion sickness in cruise ship passengers. J Travel Med. 2000;7(3):120-124.

9. Turner M, Griffin MJ, Holland I. Airsickness and aircraft motion during short-haul flights. Aviat Space Environ Med. 2000;71(12):1181-1189.

10. US Army Medical Department. Motion Sickness. Medical Aspects of Harsh Environments, Uniformed Services University of the Health Sciences. 2002;35:1048-1083.

11. Benson AJ, Ernsting K. Motion sickness in Aviation Medicine (2nd edn), Butterworths, London, England. 1988. p.318 - 338 .

12. Strongin TS, Charlton SG. Motion sickness in operational bomber crews. Aviat Space Environ Med. 1991;62(1):57-59.

13. Kennedy RS, Lilienthal MG, Berbaum KS, et al. Simulator sickness in U.S. Navy flight simulators. Aviat Space Environ Med. 1989;60(1):10-16.

14. Ungs TJ. Simulator induced syndrome in Coast Guard Aviators. Aviat Space Envir Med. 1988;59(3):267-272.

15. Sparto PJ, Whitney SL, Hodges LF, et al. Simulator sickness when performing gaze shifts within a wide field of view optic flow environment: preliminary evidence for using virtual reality in vestibular rehabilitation. Neuroengineering Rehabil. 2004;1(1):1-14.
16. Blok RJ. Simulator sickness in the Army UH-60A Blackhawk flight simulator. Mil Med. 1992;157(3):109-111.

17. Kennedy RS, Lane NE, Berbaum KS, et al. Simulator sickness questionnaire: An enhanced method for quantifying simulator sickness. Int J Aviat Psychol. 1993;3(3):203-220.

18. Kennedy RS, Drexler JM, Compton DE, et al. Configural scoring of simulator sickness, cybersickness, and space adaptation syndrome: Similarities and differences. In: Hettinger LJ \& Hass MW (Eds.), Virtual and adaptive environments: Applications, implications, and human performance issues. Lawrence Erlbaum Associates, Mahwah, New Jersey, USA. 2003. p. 247-278.

19. Solimini AG, Mannocci A, Di Thiene D, et al. A survey of visually induced symptoms and associated factors in spectators of three dimensional stereoscopic movies. BMC Public Health. 2012;12:779.

20. Ji JT, So RH, Cheung RT. Isolating the effects of vection and optokinetic nystagmus on optokinetic rotation-induced motion sickness. Hum Factors. 2009;51(5):739-751.

21. Adolfo M Bronstein, John F Golding, Michael A Gresty. Visual Vertigo and Motion Sickness. Vestibular Migraine and Related Syndromes. 2014. p.91-104.

22. Stern RM, Hu S, Anderson RB, et al. The effects of fixation and restricted visual field on vection-induced motion sickness. Aviat Space Environ Med. 1990;61(8):712-715.

23. Bernhard E Riecke, Daniel Feuereissen, John J Rieser. Auditory selfmotion illusions (circular vection) can be facilitated by vibrations and the potential for actual motion. ACM Transactions on Applied Perception (TAP) $A C M$ New York, NY. 2003;6(3).

24. Brandt T, Dichgans J, Koenig E. Differential effects of central versus peripheral vision on egocentric and exocentric motion perception. Exp Brain Res. 1973;16(5):476-491.

25. Webb NA, Griffin MJ. Optokinetic stimuli: motion sickness, visual acuity, and eye movements. Aviat Space Environ Med. 2002;73(4):351-358.

26. Krueger WW. Controlling Motion Sickness and Spatial Disorientation as Well as Enhancing Vestibular Rehabilitation with a User Worn SeeThrough Display Eyewear. Laryngoscope . 2011;121(Suppl 2):17S -35.

27. Bonato F, Bubka A, Krueger WWO. A Wearable Device Providing a Visual Fixation Point for the Alleviation of Motion Sickness Symptoms. Mil Med. 2015;180(12):1268-1272.

28. Attias JC, Gordon J, A Bolnick A, et al. Nystagmus episodes related to sea sickness. Archives of oto-rhino-laryngology. 1987;244(2):84-87.

29. Bukhtiiarov IV, Chistov SD, Ponomarenko KV, et al.Interocular asymmetry of nystagmus and motion sickness. Aviakosm Ekolog Med. 2011;45(3):34-39.

30. Krausen C, Hamann KF. Effect of stabilization of the visual field on rotatory testing. Laryngol Rhinol Otol (Stuttg). 1986;65(3):151-153.

31. Kato I, Kimura Y, Aoyagi M, et al. Visual suppression of caloric nystagmus in normal individuals. Acta Otolaryngol. 1977;83(34):245-251.

32. Wiggins $\mathrm{D}$, Woodhouse JM, Margrain $\mathrm{TH}$, et al. Infantile nystagmus adapts to visual demand. Invest Ophthalmol Vis Sci. 2007;48(5):2089-2094.

33. Pola J, Wyatt HJ, Lustgarten M. Visual fixation of a target and suppression of optokinetic nystagmus: effects of varying target feedback. Vision Res. 1995;35(8):1079-1087.

34. Watabe H, Hashiba M, Baba S. Voluntary suppression of caloric nystagmus under fixation of imaginary or after-image target. Acta Otolaryngol Suppl. 1996;525:155-157.

35. Matsangas P, McCauley ME, Becker W. The Effect of Mild Motion Sickness and Sopite Syndrome on Multitasking Cognitive Performance. Human Factors. 2014;56(6):1124-1135. 